\title{
Interval-valued grey fuzzy based groundwater remediation management under uncertainty
}

\author{
Lixia Ren $^{\mathrm{a}}$, Li He ${ }^{\mathrm{b}, *}, \mathrm{Xi}_{\text {Cheng }}^{\mathrm{c}}$ and Zheng Wang ${ }^{\mathrm{d}}$ \\ School of Renewable Energy, North China Electric Power University, China

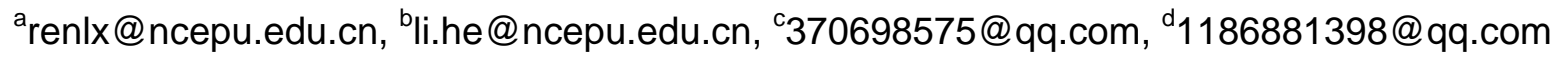 \\ ${ }^{*}$ Corresponding Author: Tel: +86-10-61772416; Fax: +86-10-61772416
}

Keywords: groundwater remediation, grey fuzzy, interval, uncertainty.

\begin{abstract}
This study is to develop an outranking method to identify compromised groundwater remediation strategies based on interval-valued grey fuzzy sets in 10-year and 20-year periods under uncertainty. The interval-valued grey fuzzy sets that could quantify the ambiguous nature of subjective judgments, have received increasing attention because of their great ability to handle imprecise and ambiguous information in real-world applications. In the case of Coal-fired Power Plant, ten alternatives and four criteria are considered. Results from the case study indicate that A8 is the optical remediation strategy under 10-year period, A2 in 20-year remediation duration.
\end{abstract}

\section{Introduction}

Because of the high cost associated with aquifer remediation processes and the potential cost reduction capabilities of optimization techniques, a large number of simulation-optimization models are proposed by planners to develop more comprehensive, complex and ambitious plans for groundwater resources systems [1, 2]. Pump and treat (PAT) is one of the established techniques (for restoring the contaminated aquifers $[3,4]$. However, many complexities and uncertainty are widely exist in designing optimal PAT systems. Thus, it is deemed necessary to develop effective optimization methods for supporting groundwater remediation management under uncertainty.

In practice, there are many optimization problems formulated using imprecise parameters (i.e. intervals and/or fuzzy number). Some researchers pointed out that it is more useful by using these two kinds of fuzzy numbers s to deal with real life problems for the following reasons, i.e. (1) they not only can represent decision maker's linguistic opinions, (2) they can represent the degrees of confidence or degrees of uncertainty of parameters [5]. Because of fuzzy, grey and uncertainty of the problems to be decided, a lot of grey fuzzy multi-criteria decision making Problems whose information of the decision making criteria may be uncertainty. A useful decision model must handle incomplete and uncertain knowledge and information.

The purpose of this paper is to develop interval-valued grey fuzzy (IGF) method to deal with these problem of multi-criteria making decisions and select the best groundwater remediation strategy from a set of candidate alternatives; ten alternatives and four criteria are considered under medium-term remediation (i.e. 10-year)and long-term remediation (i.e. 20-year).

\section{Methodology}

The theory of interval-valued grey fuzzy sets is used for modeling impressions and quantifying the ambiguous nature of subjective judgments under uncertain information. The IGF method for solving a multiple criteria decision analysis groundwater remediation problem is summarized in the following steps:

(1) Formulate a multiple criteria decision analysis groundwater remediation problem. Specify the alternative set $A=\left\{A_{1 t}, \ldots, A_{i t} \ldots A_{n t}\right\}$ and the criterion set $C=\left\{C_{1 t}, \ldots C_{j t}, \ldots\right\} . A_{i j t}$ represents the performance of action $i$ as regards to criterion $j$ for $t$ remediation period. In this paper, 10 actions and 4 evaluation criteria (i.e. total pumping volume (TPV), total cost (TC), average remaining 
contaminant concentration (ARCC), and maximum excess life time cancer risk (MELCR)) for 10-year and 20-year remediation periods are considered, respectively;

(2) Confirm interval-valued fuzzy evaluative performance and the gradation that represent the credibility form obtained information for alternative $A_{i t}$ with respect to criterion $C_{j t}$ in different $t$ remediation periods. $\tilde{A}_{\otimes i j t}=\left(\left[A_{i j t}^{L}, A_{i j t}^{U}\right],\left[v_{i j t}^{L}, v_{i j t}^{U}\right]\right)$, where $\tilde{A}_{i j t}=\left[A_{i j t}^{L}, A_{i j t}^{U}\right]$ and $A_{i j t}=\left[v_{i j t}^{L}, v_{i j t}^{U}\right]$ represent the intervals information and gradation for which alternative $A_{i t}$ is evaluated with respect to criterion $C_{j t}$, respectively.

(3) Divide $C_{j t}$ into benefit criteria (i.e., a larger value of $C_{j t}$ indicates a greater preference) and cost criteria (i.e., a smaller value of $C_{j t}$ indicates a greater preference), and then standardize the original data for $C_{j t}$;

(4) Assign the weights $w=\left\{w_{1 t}, \ldots w_{j t}, \ldots\right\}$ to the criteria $C=\left\{C_{1 t}, \ldots C_{j t}, \ldots\right\}$, which should reflect the relative importance of each criterion according to the priorities and perceptions of the decision-maker.

(5) Calculate integrated evaluated value for each alternative $A_{i t}$ with respect to criterion $C_{j t}$;

$$
\begin{aligned}
& \Phi_{i t}^{L}=\sum_{J=1}^{n}\left(A_{i j t}^{L},\left[v_{i j t}^{L}, v_{i j t}^{U}\right]\right) \cdot w_{j t}=\sum_{J=1}^{n}\left(A_{i j t}^{L} \cdot w_{j t},\left[\max \left(v_{i j t}^{L}, 0\right), \max \left(v_{i j t}^{U}, 0\right)\right]\right)=\sum_{J=1}^{n}\left(A_{i j t}^{L} \cdot w_{j t},\left[v_{i j t}^{L}, v_{i j t}^{U}\right]\right) \\
& \Phi_{i t}^{U}=\sum_{J=1}^{n}\left(A_{i j t}^{U},\left[v_{i j t}^{L}, v_{i j t}^{U}\right]\right) \cdot w_{j t}=\sum_{J=1}^{n}\left(A_{i j t}^{U} \cdot w_{j t},\left[\max \left(v_{i j t}^{L}, 0\right), \max \left(v_{i j t}^{U}, 0\right)\right]\right)=\sum_{J=1}^{n}\left(A_{i j t}^{U} \cdot w_{j t},\left[v_{i j t}^{L}, v_{i j t}^{U}\right]\right)
\end{aligned}
$$

(6) Convert interval gray fuzzy values to certain numbers;

$$
\begin{aligned}
& \left.\Phi_{i t}^{L}=\sum_{J=1}^{n}\left(A_{i j t}^{L} \cdot w_{j t},\left[v_{i j t}^{L}, v_{i j t}^{U}\right]\right)=\sum_{J=1}^{n} A_{i j t}^{L} \cdot w_{j t} \cdot\left(1-(1-\theta) \cdot v_{i j t}^{L}+\theta \cdot v_{i j t}^{U}\right]\right) \\
& \left.\Phi_{i t}^{U}=\sum_{J=1}^{n}\left(A_{i j t}^{U} \cdot w_{j t},\left[v_{i j t}^{L}, v_{i j t}^{U}\right]\right)=\sum_{J=1}^{n} A_{i j t}^{U} \cdot w_{j t} \cdot\left(1-(1-\theta) \cdot v_{i j t}^{L}+\theta \cdot v_{i j t}^{U}\right]\right)
\end{aligned}
$$

(7) Determine the complete preorder for the set $A$ of alternatives by calculating the possibility degree between $A_{i t}$ and $A_{i}$ 't and priority vector.

$$
\begin{aligned}
& p_{i i^{\prime} t}\left(A_{i t}, A_{i^{\prime} t}\right)=\frac{\min \left\{\Phi_{i t}^{U}-\Phi_{i t}^{L}+\Phi_{i^{\prime} t}^{U}-\Phi_{i^{\prime} t}^{L}, \max \left(\Phi_{i t}^{U}-\Phi_{i^{\prime} t}^{L}, 0\right)\right\}}{\Phi_{i t}^{U}-\Phi_{i t}^{L}+\Phi_{i^{\prime} t}^{U}-\Phi_{i^{\prime} t}^{L}} \\
& p v_{i t}=\frac{1}{n(n-1)}\left(\sum_{j=1}^{n} p_{i j t}+\frac{n}{2}-1\right)
\end{aligned}
$$

(8) Rank the $n$ groundwater remediation alternatives according to the descending order of their $p v_{i t}$ values. The higher $p v_{i t}$ value, the better the action.

\section{Results}

A Coal-fired Power Plant located in southeastern China, where the groundwater system has been contaminated because of the spill and leakage of pollutants in the ash field is applied to demonstrate the performance of the IGF method for identifying the most desirable groundwater remediation alternative. A PAT system was applied to eliminate major contaminants. Fourteen wells (i.e. two injection, four extraction wells and eight monitoring wells) compose the PAT system for groundwater remediation. Two remediation periods are considered, including 5, 10, and 15 years to represent medium and long remediation periods.

The detailed evaluation of each alternatives for the four criterions for 10-year remediation durations are calculated as shown in Table 1, respectively. TPV it is the total pumping volume for all injection/extraction wells during each period $\left(10^{6} \mathrm{~m}^{3}\right)$. TC is the total costs that invested for solving groundwater pollution remediation problem in the study site $\left(10^{6} \$\right)$. ARCC is the average remaining contaminant concentration in each monitoring well after PAT system $(\mu \mathrm{g} / \mathrm{L})$. MELCR is maximum excess life time cancer risk which could measure the degree of human health risks.

Table 1 Performance of each alternative action during 10-year period 


\begin{tabular}{lllll}
\hline Action & TPV & TC & ARCC & MELCR \\
\hline \multirow{2}{*}{ A1 } & $([0.855,1.161]$, & $([2.217,4.118]$, & $([0.542,1.006]$, & $([3.988,5.118]$, \\
& {$[0.1,0.2]$} & $[0.3,0.5])$ & $[0.3,0.5])$ & $[0.4,0.6])$ \\
A2 & $([0.524,0.712]$, & $([1.359,2.524]$, & $([2.055,3.816]$, & $([7.548,9.686]$, \\
& {$[0.2,0.3]$} & $[0.3,0.4])$ & $[0.4,0.5])$ & $[0.5,0.6])$ \\
A3 & $([0.593,0.805]$, & $([1.538,2.856]$, & $([1.425,2.647]$, & $([5.938,7.62]$, \\
& {$[0.2,0.4]$} & $[0.4,0.5])$ & $[0.4,0.5])$ & $[0.3,0.5])$ \\
A4 & $([1.2,1.63]$, & $([3.111,5.778]$, & $([0.561,1.042]$, & $([3.158,4.053]$, \\
& {$[0.1,0.2]$} & $[0.3,0.4])$ & $[0.2,0.4])$ & $[0.4,0.5])$ \\
A5 & $([1.104,1.499]$, & $([2.861,5.313]$, & $([0.223,0.415]$, & $([0.927,1.19]$, \\
& {$[0.1,0.2]$} & $[0.2,0.3])$ & $[0.3,0.4])$ & $[0.4,0.6])$ \\
A6 & $([1.117,1.517]$, & $([2.897,5.38]$, & $([0.473,0.879]$, & $([4.11,1.166]$, \\
& {$[0.2,0.3]$} & $[0.4,0.5])$ & $[0.3,0.5])$ & $[0.3,0.4])$ \\
A7 & $([1.214,1.648$, & $([3.147,5.845]$, & $([0.452,0.84]$, & $([2.709,3.477]$, \\
& {$[0.1,0.2]$} & $[0.2,0.3])$ & $[0.4,0.5])$ & $[0.4,0.5])$ \\
A8 & $([1.035,1.405]$, & $([2.682,4.981]$, & $([0.225,0.417]$, & $([0.901,1.156]$, \\
& {$[0.1,0.3]$} & $[0.3,0.4])$ & $[0.2,0.3])$ & $[0.3,0.4])$ \\
A9 & $([1.2,1.63]$, & {$[3.111,5.778]$,} & $([0.685,1.271]$, & $([5.97,7.662]$, \\
& {$[0.1,0.2]$} & $[0.2,0.5])$ & $[0.3,0.4])$ & $[0.4,0.6])$ \\
A10 & $([1.145,1.555$, & $([2.968,5.512]$, & $([0.638,1.184]$, & $([5.392,6.919]$, \\
& {$[0.1,0.2]$} & $[0.2,0.3])$ & $[0.3,0.5])$ & $[0.2,0.5])$ \\
\hline
\end{tabular}

These weights for each remediation periods are determined based analytical hierarchy process (AHP) method which is the best known and most widely used in MCDA approach, as well as the preference of the related experts and stakeholders, as shown in Table 2. Compared with that of 10-year period, the weight of and TPV and TC has been increased from 0.167 to 0.321 and 0.271 to 0.411 , respectively, which means that TPV and TC have higher priority than others by decision maker because remediation of PAT system is a costly process.

Table 2 Weight for each criterion

\begin{tabular}{lllll}
\hline Weight & TPV & TC & ARCC & MELCR \\
\hline $\begin{array}{l}\text { 10-yea } \\
\text { r }\end{array}$ & 0.167 & 0.271 & 0.243 & 0.318 \\
20-yea & & & & \\
r & 0.321 & 0.411 & 0.127 & 0.141 \\
\hline
\end{tabular}

Table 3 and 4 shows the possibility degree between two actions for each periods, respectively. If $p_{i^{\prime} t}\left(A_{i t}, A_{i^{\prime} t}\right)=1$, which indicates $A_{i t}$ is strict higher than $A_{i^{\prime} t}$ in criterion during $t$ period. If $p_{i i^{\prime} t}\left(A_{i t}, A_{i^{\prime} t}\right)=0$, which indicates $A_{i^{\prime} t}$ is strict higher than $A_{i t}$ in criterion during t period.

Table 3 Possibility degree between two actions during 10-year period

\begin{tabular}{lllllllllll}
\hline$p$ & 1 & 2 & 3 & 4 & 5 & 6 & 7 & 8 & 9 & 10 \\
\hline 1 & 0.498 & 0.438 & 0.517 & 0.537 & 0.130 & 0.332 & 0.498 & 0.032 & 0.654 & 0.580 \\
2 & 0.560 & 0.501 & 0.580 & 0.599 & 0.199 & 0.450 & 0.562 & 0.102 & 0.712 & 0.641 \\
3 & 0.478 & 0.419 & 0.498 & 0.517 & 0.110 & 0.294 & 0.478 & 0.012 & 0.636 & 0.561 \\
4 & 0.462 & 0.402 & 0.482 & 0.501 & 0.090 & 0.257 & 0.462 & 0.000 & 0.623 & 0.547 \\
5 & 0.868 & 0.802 & 0.889 & 0.910 & 0.500 & 0.917 & 0.874 & 0.403 & 1.000 & 0.947 \\
6 & 0.663 & 0.550 & 0.702 & 0.742 & 0.082 & 0.496 & 0.670 & 0.000 & 0.979 & 0.825 \\
7 & 0.498 & 0.437 & 0.518 & 0.537 & 0.125 & 0.326 & 0.498 & 0.026 & 0.658 & 0.582 \\
8 & 0.964 & 0.898 & 0.985 & 1.000 & 0.597 & 1.000 & 0.972 & 0.500 & 1.000 & 1.000 \\
9 & 0.342 & 0.287 & 0.360 & 0.377 & 0.000 & 0.017 & 0.339 & 0.000 & 0.498 & 0.423 \\
10 & 0.416 & 0.359 & 0.435 & 0.453 & 0.053 & 0.174 & 0.415 & 0.000 & 0.573 & 0.498 \\
\hline
\end{tabular}


Table 4 Possibility degree between two actions during 20-year period

\begin{tabular}{lllllllllll}
\hline$p$ & 1 & 2 & 3 & 4 & 5 & 6 & 7 & 8 & 9 & 10 \\
\hline 1 & 0.501 & 0.342 & 0.403 & 0.718 & 0.376 & 0.505 & 0.500 & 0.460 & 0.639 & 0.498 \\
2 & 0.660 & 0.500 & 0.563 & 0.873 & 0.522 & 0.656 & 0.653 & 0.612 & 0.794 & 0.652 \\
3 & 0.597 & 0.438 & 0.500 & 0.812 & 0.464 & 0.596 & 0.593 & 0.552 & 0.733 & 0.592 \\
4 & 0.281 & 0.127 & 0.185 & 0.498 & 0.184 & 0.300 & 0.293 & 0.256 & 0.424 & 0.289 \\
5 & 0.627 & 0.481 & 0.538 & 0.819 & 0.502 & 0.625 & 0.622 & 0.585 & 0.749 & 0.621 \\
6 & 0.494 & 0.343 & 0.402 & 0.697 & 0.376 & 0.498 & 0.494 & 0.456 & 0.624 & 0.492 \\
7 & 0.501 & 0.347 & 0.407 & 0.708 & 0.380 & 0.505 & 0.500 & 0.462 & 0.633 & 0.498 \\
8 & 0.538 & 0.387 & 0.445 & 0.740 & 0.416 & 0.540 & 0.536 & 0.498 & 0.667 & 0.534 \\
9 & 0.361 & 0.208 & 0.266 & 0.574 & 0.254 & 0.374 & 0.368 & 0.331 & 0.500 & 0.365 \\
10 & 0.501 & 0.347 & 0.407 & 0.708 & 0.380 & 0.505 & 0.500 & 0.462 & 0.633 & 0.498 \\
\hline
\end{tabular}

Table 5 presents the priority vector of possibility degree matrices and ranking for each action in different periods. As we can see form the table, the complete preorder during each remediation periods is different. A8 and A2 is the optical remediation strategy under 10-year and 20-year period, respectively. The prefer ability of A8 or A2 over the other alternatives is significant.

Table 5 Priority vector of possibility degree matrices and ranking for each action

\begin{tabular}{lllllllllll}
\hline Ranking & 1 & 2 & 3 & 4 & 5 & 6 & 7 & 8 & 9 & 10 \\
\hline \multirow{2}{*}{ 10-year } & A8 & A5 & A6 & A2 & A1 & A7 & A3 & A4 & A10 & A9 \\
& 0.144 & 0.135 & 0.108 & 0.099 & 0.091 & 0.091 & 0.089 & 0.087 & 0.082 & 0.074 \\
\hline \multirow{2}{*}{ 20-year } & A2 & A5 & A3 & A8 & A1 & A7 & A10 & A6 & A9 & A4 \\
& 0.117 & 0.113 & 0.110 & 0.103 & 0.099 & 0.099 & 0.099 & 0.099 & 0.084 & 0.076 \\
\hline
\end{tabular}

\section{Summary}

This paper presented interval-valued grey fuzzy method applied a Coal-fired Power Plant to identify the best groundwater remediation strategy from a set of candidate alternatives. In this study, ten alternatives and four criteria in 10-year and 20-year remediation periods were considered, where certain weights of criteria and the criteria values in the forms of interval grey fuzzy numbers are introduced. The process of integrated evaluated value, interval gray fuzzy values into certain numbers, possibility degree and complete preorder are involved. Results showed that A8 is determined as the compromised choice during 10-year remediation period, A2 in 20-year.

\section{Acknowledgements}

This research was supported by the China National Funds for Excellent Young Scientists (51422903), National Natural Science Foundation of China (41271540), Program for New Century Excellent Talents in University of China (NCET-13-0791), and Fundamental Research Funds for the Central Universities.

\section{References}

[1] M.J. Kazemzadeh-Parsi, F. Daneshmand, M.A. Ahmadfard, J. Adamowski, R. Martel, Optimal groundwater remediation design of pump and treat systems via a simulation-optimization approach and firefly algorithm, Eng. Optimiz. 47 (2015) 1-17.

[2] U.S. McKnight, M. Finkel, A system dynamics model for the screening-level long-term assessment of human health risks at contaminated sites, Environ Modell Softw. 40 (2013) 35-50.

[3] M. Meenal, T.I. Eldho, Simulation-optimization model for groundwater contamination remediation using meshfree point collocation method and particle swarm optimization. Sadhana 37 (2012) 351-369. 
[4] N.Y. Ko, K.K. Lee, Y. Hyun, Optimal groundwater remediation design of a pump and treat system considering clean-up time, Geosci J 9 (2005) 23-31.

[5] S.J. Chen, S.M. Chen, A New Information Fusion Method Based on Interval-Valued Fuzzy Numbers for Handling Multi-Criteria Fuzzy Decision-Making Problem, IEEE International Conference on Fuzzy Systems (2004) 825-830. 